\title{
Dynamic Analysis of Alternative Elements in an Automated Packaging System Based on 5G Internet of Things
}

\author{
Jian Long (iD \\ School of Art \& Design, Guilin University of Electronic Technology, Guilin 541000, China \\ Correspondence should be addressed to Jian Long; lomjian@guet.edu.cn
}

Received 6 December 2021; Revised 28 December 2021; Accepted 3 January 2022; Published 28 January 2022

Academic Editor: Gengxin Sun

Copyright (c) 2022 Jian Long. This is an open access article distributed under the Creative Commons Attribution License, which permits unrestricted use, distribution, and reproduction in any medium, provided the original work is properly cited.

\begin{abstract}
The arrival of 5G will usher in an era of "Internet of Everything." Massive Internet of Things data contains great value in the dynamic analysis of alternative elements of automated packaging systems. From the perspective of the realization of personalized customization functions, this article solves the problem of dynamic analysis of alternative elements in the automated packaging system. We analyze the connection mechanism and interaction method between the cloud service system layer and the mobile terminal service layer, and carry out the corresponding software design. From the perspective of the realization of the intelligent production of the system in this paper, this topic mainly studies the information interaction mechanism and production control mechanism of the cloud service system and the manufacturing system. Based on the hardware of the manufacturing system layer, a flexible production implementation mechanism is formulated to make it the basis for the implementation of intelligent production of the system. Based on the massive data processing capabilities of the cloud service system, the information processing mechanism and the production planning decision-making mechanism are formulated for it, so as to realize the intelligent adjustment of the manufacturing system layer in the production process. For the connection scenario of IoT group paging, based on the application of NB-IoT technology in the next-generation mobile communication network, the focus of network optimization is to ensure the random access performance of IoT devices as much as possible. To this end, this paper proposes a random access optimization strategy for IoT group paging based on time slot scattering. We establish a mathematical model based on queuing theory for the connection scenario of the Io $\mathrm{T}$ group paging, then use the mathematical formula to derive the number of IoT devices scattered to each time slot in the initial state, thereby deriving the specific time slot scattering algorithm. This paper establishes a list of credit nodes, changes the participation mode of consensus nodes from static to dynamic, and supports voting to select trusted nodes. We designed a credit evaluation mechanism as a basis for consensus node elections to improve system's fault tolerance rate. The algorithm process was simplified, and the PBFT algorithm process was simplified from a 3-phase protocol to a 2-phase protocol to further reduce communication bandwidth overhead and algorithm time. Simulation analysis shows that, compared with the PBFT algorithm, the proposed algorithm improves node flexibility and fault tolerance while reducing communication bandwidth overhead by about $45 \%$, packaging throughput by about $4 \%$, and latency by about $3 \%$.
\end{abstract}

\section{Introduction}

The Internet of Things is known as the third revolutionary wave of innovation in the information industry after computers and the Internet [1]. Driven by such a wave, any object in the world is connected to the Internet according to a preagreed agreement, and communications and information exchange services are provided [2]. The research on the architecture of the information and communication technology Internet of Things information service system represented by the Internet of Things is developing rapidly at an unprecedented speed, greatly affecting world's technological landscape [3]. Under the service of $5 \mathrm{G}$ network, the amount of data information that the Internet of Things information service system needs to serve in the future will increase exponentially. According to HIS statistics, by 2025 , there will be 150 billion wireless terminal devices connected to the network worldwide, among which the wireless terminal devices connected to the Internet of Things will reach about 85 billion [4]. By then, more than 90\% of 
world's wireless terminal devices need to seek services in the Internet of Things information service network. In the future, the number of mobile terminals and Internet of Things terminal devices will far exceed the total population of the world. The Internet of Things information service network will carry a large amount of data traffic growth [5]. Faced with such a huge demand for data services, wireless terminal equipment directly connected to the network has large interference, high power consumption, low spectrum utilization of system communication links, inability to achieve compatibility between mobile terminals and IoT terminals, and inability to integrate into the Internet application ecosystem. The problem is becoming more and more obvious.

The development of the packaging industry requires continuous research and development of more advanced packaging technologies to meet the ever-increasing demand of market competition, and the innovation of packaging technology is more reflected in the degree of intelligence of packaging equipment [6]. Therefore, the design and development of a more economical and smarter new packaging system is of great significance to the development of the packaging industry [7]. This topic fully considers the actual needs of manufacturers and the packaging market and ensures that the packaging process is simple; the equipment is stable and easy to operate while ensuring that the system is fully functional. From the perspective of the enterprise, the design of the packaging system solves the problems of high packaging cost, low efficiency, and poor precision from the root and effectively improves the market competitiveness of the enterprise [8]. From an industry perspective, the design of the packaging system provides a good reference platform for the research and development of automatic packaging technology for small rubber products, fills the current technical gap in automatic packaging for small products, and promotes the rapid development of the packaging industry [9]. At present, the labor cost of manufacturing transformation and upgrading is increasing year by year, and the degree of automation and informationization in the packaging field is low [10]. This article combines mainstream packaging methods and selects objects to be packaged for research. This article can improve the competitiveness of enterprises, liberate people from simple and repetitive labor, and can reduce production costs and improve the controllability of product quality. Therefore, studying the dynamic analysis of alternative elements in the automated packaging system has important theoretical and practical significance.

This article summarizes the overall functional requirements and implementation basis of the automated packaging system for personalized customization of this topic, carries out a structured and layered design, and at the same time, gives the basic architecture and platform of each level of system's subsystems. Through the introduction of these platforms and technologies, the platform foundation of "intelligence," "flexibility," and "personalized service" to be realized in the case design of this article is completed. This paper analyzes the limitations of NB-IoT cellular sites when processing group paging IoT device connection requests, and then, in the IoT group paging model based on queuing theory, studies the random access optimization of the scattering of the arrival rate of the IoT devices in the scattered group paging plan. In order to obtain the interval value of the time slot scattering, the upper limit of the arrival rate in each RA-slots in the random access process is studied, and starting from the expected IoT arrival rate, according to the current number of IoT devices, iteratively, we calculate each RA-slot initial IoT arrival rate and then get the IoT slot scattering algorithm. At the same time, according to the maximum processing capacity of the current NBIoT cell site, the critical value for starting the IoT time slot scattering algorithm is given. This paper establishes a list of credit nodes and improves the participation mode of consensus nodes from static to dynamic, and nodes can enter or exit dynamically. A node credit evaluation mechanism is designed to follow the consensus node election and improve system's fault tolerance rate. Based on the credit evaluation mechanism, the three-phase protocol of the PBFT algorithm is simplified into two phases, which further reduces communication overhead and algorithm delay.

\section{Related Work}

Compared with the previous generations of mobile communication technologies, the biggest difference of the $5 \mathrm{G}$ system is that it does not reform and upgrade the key technologies used in the previous communication systems, but integrates many technologies to make them complement each other and play their respective strengths. The rapid growth of network service traffic and the number of devices connected to the network will make the spectrum resources in the future communication network more tense. Although some new spectrum resources will be unearthed, the real key is to allocate the existing spectrum resources reasonably to maximize the value of the limited resources.

The packaging industry is a service-oriented manufacturing industry. It is the last process in enterprise production [11]. It is a necessary link for all manufacturing engineering to make finished products. It is an inaccessible part of the manufacturing process. Therefore, automated packaging is very important for enterprises. With the development of robotics, vision technology, modern control technology, computer technology, artificial intelligence technology, and material technology, automated packaging has emerged [12]. Automated packaging not only improves labor production efficiency, reduces product costs, and enhances the competitiveness of enterprises. Moreover, the packaging quality can be more controllable, and at the same time, a lot of work injuries can be reduced [13]. As more functions are integrated in the automated packaging system, automated packaging is more favored by enterprises, such as anticounterfeiting technology, intelligent sorting systems, and intelligent detection systems [14].

At present, automated packaging is used in beverages, food, medicine, nonfood industrial products and industrial products [15]. Automated packaging is developing in terms of multiple functions, aesthetics and personalization, light and smart equipment, and material saving. As the automated packaging industry in foreign countries started earlier 
than the domestic development, packaging in Western countries has been fully automated. In the packaging field, there are famous companies such as ABB, EPSON, KUKA, Omron, and FANUC [16].

Packaging equipment control technology refers to an advanced technology in which packaging equipment can complete packaging work in accordance with a preplanned work flow without human intervention. At present, most of the truss packaging equipment at home and abroad adopts a distributed control method with programmable logic control technology as the main and embedded control technology as the supplement [17]. On the one hand, it can retain the advantages of programmable logic control technology in an industrial environment with more stable performance, better safety, and better compatibility with electrical equipment; on the other hand, it can ensure that the system has better economic efficiency and applicability.

Relevant scholars apply Pro/E behavioral modeling technology to the optimization of packaging machinery parts, by modeling the drive mechanism of the plug-in plate and establishing measurement features, inserting motion analysis features and relationship analysis features, and performing sensitivity analysis to obtain the optimal parameters, and then improve the design efficiency of packaging machinery [18]. Relevant scholars have conducted algorithmic research on the packing problem of irregular objects and put forward some constructive algorithms that can solve various twodimensional packing problems [19]. He proposed several integer programming models to determine the degree of association between irregular parts and packaging boxes and then established a mixed integer programming model to solve the problem of packing irregular parts into packaging boxes.

Swiss ABB's robotic automated packaging is mainly used in three aspects: picking, packaging, and palletizing [20-22]. In terms of picking technology, ABB's picking solutions are used in many types of products, applications, and packaging lines. It includes various industries such as frozen food, bread candy, ice cream, meat and fish products, cheese, pet food, medical supplies, shampoo bottles, and perfume bottles $[23,24]$. In the picking solution, there are subdivisions for different scenarios. Aiming at high-precision picking and unloading operations, $\mathrm{ABB}$ currently launches the IRB360 FlexPicker TM second-generation triangular robot solution [25]. For applications that need to achieve six-axis flexibility, require slightly lower cycle time, and have a payload of no more than $5 \mathrm{~kg}, \mathrm{ABB}$ has launched the IRB 140 robot solution; in the packaging industry, $\mathrm{ABB}$ has launched the IRB260 robot solution. The packaging system is equipped with $\mathrm{ABB}$ 's unique visual assisted conveyor tracking system to maintain continuous product passing and rapid packaging [26, 27]. ABB also provides a series of high-speed bag stacking, box stacking, and palletizing robots [28].

\section{Methods}

3.1. 5G Private Network Networking Technology. According to the evolution of the network architecture, 3GPP has defined two networking modes for $5 \mathrm{G}$ services: nonindependent networking NSA and independent networking SA. NSA uses $4 \mathrm{G}$ base stations as control plane anchor points to access $4 \mathrm{G}$ core network or $5 \mathrm{G}$ core network, that is, use existing $4 \mathrm{G}$ infrastructure for $5 \mathrm{G}$ network deployment. NSA is a transitional solution that can only support Ultra Mobile Broadband (e MBB) services, and most 5G features cannot be realized. At this stage, $5 \mathrm{G}$ services for public users will run in NSA mode; SA is a standard $5 \mathrm{G}$ networking mode. 5G base stations are used as control plane anchor points to access the $5 \mathrm{G}$ core network. 5G's new core technologies, such as end-to-end slicing, can support various innovative business operations in the SA mode and meet the guaranteed service-level agreements (SLA) for different businesses.

The 5G network architecture mainly includes two parts, the $5 \mathrm{G}$ core network $5 \mathrm{GC}$ and the radio access network NGRAN. Currently, the NSA networking mode is adopted, and it is evolving to the SA networking mode. The $5 \mathrm{G}$ core network mainly has three functional logical network elements or virtual network elements to undertake, namely, UPF, $\mathrm{SMF}$, and AMF. The $5 \mathrm{G}$ radio access network includes two types of network elements: gNB and ng-eNB, where gNB provides NR user plane and control plane functions and protocols, and ng-eNB provides E-UTRA user plane and control plane functions and protocols. Among the two main interfaces, NG belongs to the interface between the wireless access network and the core network, and Xn belongs to the interface between wireless network nodes.

3.2. $5 G$ Mobile Edge Computing. The deployment locations of mobile edge computing nodes are mainly divided into three types: edge level, location level, and regional level. The three have different characteristics, respectively. Among them, the transmission delay is edge level<location level<regional level; the overall transmission bandwidth is regional level $>$ location level $>$ edge level. To determine the specific location of the mobile edge computing node deployment, it needs to be determined according to the specific service type, the specific characteristics, and requirements of the service scenario.

Mobile edge computing (MEC) refers to the deployment of IT service environments and computing capabilities on the edge of the network closer to users or data sources and provides users with a call interface for underlying communication services. In the $4 \mathrm{G}$ era, operators and equipment manufacturers have initiated MEC technical research, product development, and live network deployment. $5 \mathrm{G}$ is a new generation of mobile communication technology proposed in response to the rapid increase in data traffic, massive device connections, and deep industry integration brought about by the development of the mobile Internet and the Internet of Things. It becomes a native capability in 5G and plays an important role in the three major application scenarios of $5 \mathrm{G}$ (e MBB, mMTC, and URLLC). At present, on a global scale, MEC has become a focus of attention and research in the industry. In various links such as technical standardization, operator 5G planning, and manufacturer 5G equipment product development, mobile edge 
computing is regarded as an important and indivisible component of $5 \mathrm{G}$ networks. Mobile edge computing, together with core functions such as network slicing, serve as an important foundation for global operators to provide services to users in the $5 \mathrm{G}$ era.

$5 \mathrm{G}$ edge computing is a multilevel edge computing technology system deployed in scenarios. The system is oriented to the diverse needs of intelligence, deploying edge computing nodes on the base station side, base station convergence side, or core network edge side to provide a variety of intelligent network access and high-bandwidth, low-latency network bearer. It relies on open and reliable connection, computing, and storage resources to support the flexible carrying of multiecological services on the access edge side. Facing the application needs of medical scenarios, smart medical multilevel edge computing provides basic capabilities such as massive terminal management, high-reliability and low-latency networking, hierarchical quality assurance, real-time data computing and cache acceleration, application container services, and network capability opening. Based on the smart medical multilevel edge computing system, operators can provide real-time, reliable, intelligent, and ubiquitous end-to-end services for smart medical.

\subsection{Overall Functional Architecture of the Automated} Packaging System. The functional architecture of the personalized customization-oriented automated packaging system is shown in Figure 1. The system architecture is divided into three levels of subsystems according to executive functions. The definitions of these three levels of subsystems from top to bottom are as follows: (1) mobile terminal service layer is the service part of the application of information interaction between people and the system, responsible for the execution of information interaction functions between customers or administrators using mobile terminal equipment and the system; (2) cloud service system layer is responsible for the reception and transmission of business information and the execution of related information processing functions for production scheduling; (3) manufacturing system layer is the production execution part of the entire system; it is responsible for product processing and manufacturing within the business scope of the enterprise.

\section{(1) Mobile terminal service layer}

The mobile terminal service layer is the user-end application access layer of the automated packaging system, which provides methods and tools for receiving user service information. With the rapid development of communication technology, various services of mobile terminal products have begun to occupy people's daily lives. With the advent of the $4 \mathrm{G} / 5 \mathrm{G}$ network era, the network transmission rate is no longer the bottleneck of mobile communication network information transmission. With the expansion of cloud computing applications, mobile cloud services (MBaaS) have become a way to connect mobile applications to cloud services.

We connect mobile applications to the back-end cloud storage by using a unified application programming inter- face (API). At present, people can use mobile terminals to complete various activities such as information inquiry, shopping, entertainment, learning, and sports. Therefore, this article combines mobile terminal services in the design of intelligent manufacturing systems to complete the mobile information interaction between humans and automated packaging systems.

Relying on mobile communication technology to achieve flexible information interaction between humans and automated packaging systems is a good choice. That is, smart phones are connected to the cloud through the mobile Internet, and the corresponding APP provided by the enterprise is used to realize the remote operation of the internal resources of the factory. Intelligent matching provides users with convenient and friendly interaction methods, so as to achieve the most direct and personalized information interaction anytime, anywhere, and then, realize the mobile terminal service layer of the automated packaging system for personalized application and management needs.

\section{(2) Cloud service system layer}

The cloud service system layer, as the information hub center of the automated packaging system, must possess the key features of computing, communication, and control integrated by the Cyber-Physical System (CPS). The cloud service system layer provides data interaction, storage, and analysis methods and interfaces for heterogeneous resource management existing in the industrial environment through cloud computing technology, web service technology, etc. and provides corresponding data computing functions. The terminal service layer and the manufacturing system layer are interrelated to form a unified and coordinated whole, so as to realize the information integration and resource sharing of the entire system.

The cloud service system layer realizes that the cloud receives personalized order information and query instructions from customer's mobile terminal through the data channel interface provided by the web service and performs order classification and integration processing on the backend server. According to the characteristics of the query instruction, the corresponding product information is mined, and then, the information processing result is pushed to the corresponding client terminal to complete the information interaction task between the client and the smart factory.

The cloud service system layer provides a data channel interface to receive equipment status information from the manufacturing system layer. According to the result of customer order integration, the decision-making information of the corresponding production plan is formulated and then issued to the control module center of the workshop production equipment. In order to facilitate the production management of the workshop manager, the cloud service system layer needs to organize the historical data information of all orders and the status information of the workshop production unit, so as to provide functions such as workshop status monitoring, equipment startup and shutdown, and raw material inventory forecasting. 


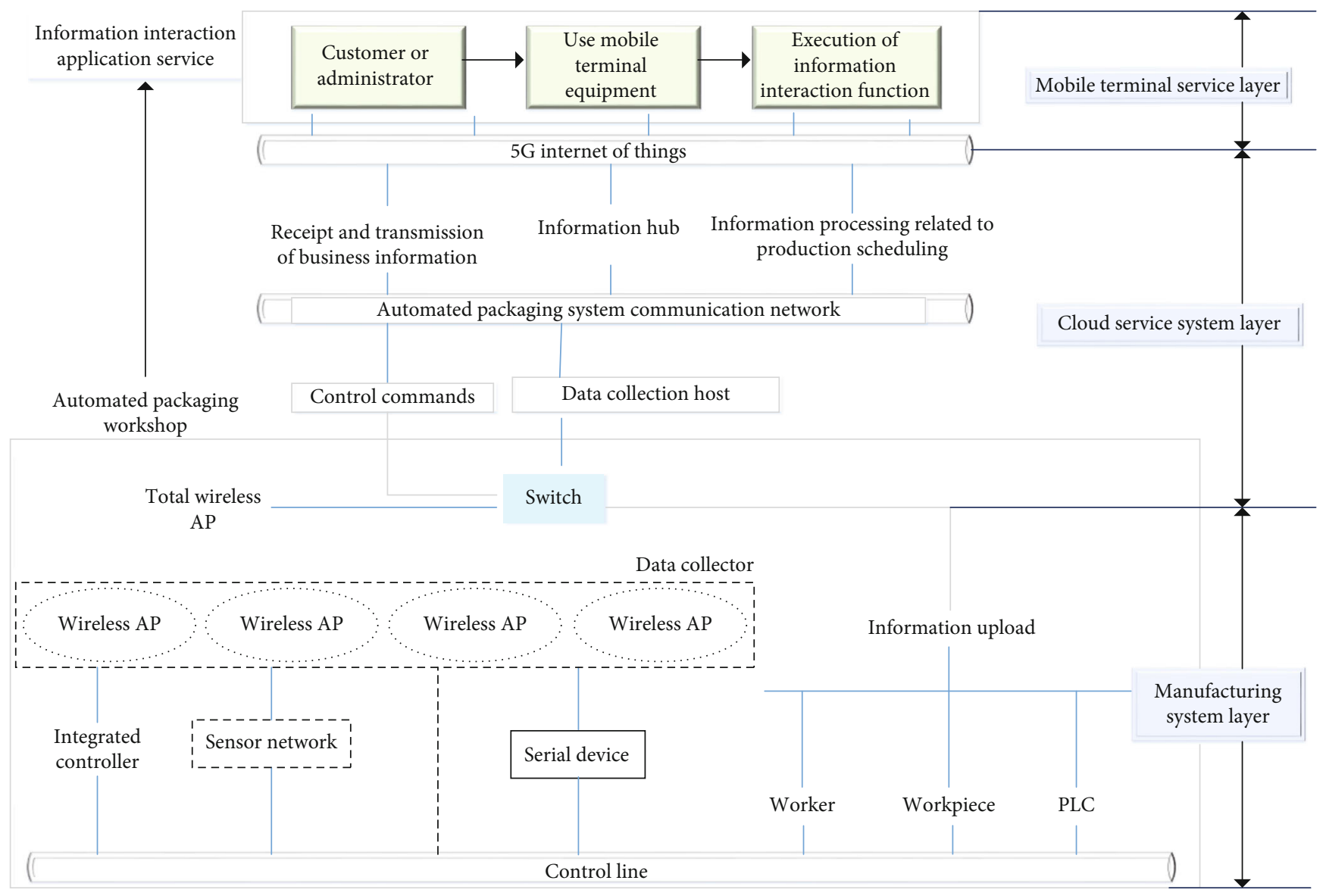

FIgURE 1: System architecture diagram.

(3) Manufacturing system layer

As the production execution part of the automated packaging system, the manufacturing system layer needs to have execution functions that can be automated and intelligent. Therefore, the manufacturing system layer needs to associate various manufacturing-related processing equipment and sensors and other components for flexible design to realize the coordinated adjustment of workshop production units. Collaborative production between workshop production units needs to be considered from two design perspectives at the manufacturing system level, namely, IoT design and reconfigurable design.

In terms of IoT design, it mainly relies on sensor networks, radio frequency, and other related industrial IoT technologies to connect discrete processing equipment, sensor components, and product parts in the factory workshop. We use sensor technology to detect the operating status of the equipment to realize the calibration and tracking of the workstation information of the processing object; rely on communication methods such as WiFi, ZigBee, and Ethernet to connect the items, processing units, cloud platforms, so as to solve the interoperability problem caused by the heterogeneity of resources in the production process.

In the reconfigurable design, the equipment and components that can complement each other's functions and can complete a set of production operations in the workshop are modularized and packaged to form a relatively independent production and processing unit. Each such independent unit is connected with other independent units by means of corresponding communication, so that they can cooperate with each other to complete industrial production tasks. The cloud service system is used as the instruction decision center for all independent production and processing units in the workshop, so as to realize the production planning and ordering of the workshop. This realizes that all equipment of the manufacturing system can adjust the working mode by itself and then quickly respond to the small batch and diversified production needs of the product.

3.4. Optimization of Random Access for IoT Group Paging Based on Queuing Theory. Under the NB-IoT cell site group paging mechanism, the service requests of IoT devices with the same GID are consistent, and the delay requirements are not high. Suppose that after the NB-IoT cell site initiates a group paging, it only processes access requests for IoT services with a certain GID in one RA-slots, and IoT devices with the same GID will initiate the same in the first RAslots access request. Therefore, the number of access requests initiated by IoT devices in I RA-slots is different, but it will decrease with the increase of time. Considering the processing capacity of NB-IoT cellular sites, it is 
necessary to scatter a large number of IoT devices on different RA-slots in order to control the number of IoT devices that initiate access requests in each RA-slots. The number of IoT devices requested for access is the same.

We define the connection rate for the first access in a RA-slots $\theta_{\mathrm{RA}}(\lambda)$ as the arrival rate in the current RA-slots multiplied by the probability of the first access success, then:

$$
\theta_{\mathrm{RA}}(\lambda)=(1-\lambda) e^{-\lambda / 2 R}
$$

Obviously, the value of $\theta_{\mathrm{RA}}$ changes with the change of the parameter $\lambda$, and the maximum value of $\theta_{\mathrm{RA}}$ can be obtained by seeking its extreme value:

$$
\theta_{\max }=(1+R) \bullet e^{-\lambda}
$$

Through analysis, it can be known that the NB-IoT cellular site system can achieve the largest first-time successful connection rate when the value of $\lambda$ is $R$. In order to keep the NB-IoT cellular site at a relatively high connection performance in I RA-slots, the arrival rate of access requests in $I_{\mathrm{RA}}$-slots must be scattered control processing, and the scattered interval value is equal to $R$.

Define $\lambda_{\mathrm{TH}}$ as the maximum capacity of the current NBIoT cellular site to handle massive IoT traffic services, then:

$$
\lambda_{\mathrm{TH}}=1-\lambda=e^{-u} .
$$

Regardless of the processing capacity of the current NBIoT cellular site, as long as there are more than one IoT devices initiating access requests in each RA-slots, theoretically, the IoT conflicts in Msg1 always exist. For each RAslots, the number of IoT devices that fail to access in the traditional LTE random access model will randomly select a RA-slots within the random back-off window to initiate an access request again, in the random access strategy for IoT group paging based on time slot scattering proposed in this chapter. Since the number of IoT devices allocated for the first time in each RA-slots is the same, the IoT devices that fail to access will randomly back off to the total access time. A certain RA-slots in the slot initiates an access request again.

Assuming that the initial arrival rate set of each RA-slot in the total access slot $I$ is represented by EN, when the random access request of an IoT device $n$ in the $i$ th RA-slot fails, the IoT device $n$ should be in $\left[i+1, I_{\mathrm{TH}}\right]$ randomly selecting an access slot $k$ as the RA-slot for reinitiating random access request. Therefore, the initial arrival rate and actual arrival rate set of each RA-slot have the following inequality relationship:

$$
\begin{cases}\lambda_{1}=\lambda_{\mathrm{TH}}=\varepsilon_{1}, & i=1, \\ \lambda_{i}=\lambda_{\mathrm{TH}}>\varepsilon_{i}, & i=2,3, \cdots, I-1, I .\end{cases}
$$

Obviously, with the exception of the first RA-slot, each RA-slot in the total access slot $I$ will be equally probabilistically selected by the IoT device that failed to access in the previous RA-slots as its new initiator. Therefore, the rela- tionship expression between the initial arrival rate and the actual arrival rate set can be further obtained:

$$
\begin{cases}\lambda_{1}=\lambda_{\mathrm{TH}}=\varepsilon_{1}, & i=1, \\ \lambda_{i}=1-\varepsilon_{i}+\prod_{k=1}^{i-1} k \lambda_{k}\left(1-e^{R \lambda_{k}}\right), & i=2,3, \cdots, I-1, I .\end{cases}
$$

The expression of the total access times $\Omega$ under the total access time slot $I$ is as follows:

$$
\Omega=(N-1)(I-1) \lambda_{\mathrm{TH}} e^{-R \lambda_{\mathrm{TH}}} .
$$

When the value of $\sigma_{I}$ appears, the $E^{N}$ set greater than the access time slot $\sigma_{I}$ is set to 0 , that is:

$$
E^{N}=\left(\begin{array}{llllll}
\varepsilon_{1} & \varepsilon_{2} & \varepsilon_{3} & \cdots & \varepsilon_{\sigma_{I}} & 0
\end{array}\right)
$$

$I_{\mathrm{TH}}$ is the time slot scattering threshold, and its mathematical expression is as follows:

$$
I_{\mathrm{TH}}=(N-1) R \lambda_{\mathrm{TH}} \cdot
$$

When $I_{\mathrm{TH}}<1$, the number of IoT devices does not exceed the maximum processing capacity of the NB-IoT cellular site. At this time, there is no need to perform actual scattering processing on the IoT devices, just initiate an access request according to the traditional LTE random access process. The system flow chart of the random access optimization strategy for IoT group paging based on time slot scattering is shown in Figure 2.

\section{Simulation Analysis}

4.1. Throughput and Delay Analysis. In order to evaluate the throughput and latency of the IOT group paging random access optimization algorithm, this article is configured with an Intel Core i7-6700M @3.40 GH processor and 16 G memory, installed on a PC with a 64-bit Windows7 system, through the Eclipse2020 platform. The PBFT and IOT group paging random access optimization algorithm was implemented by programming in Java language, and the delay and throughput test were carried out. Among them, the implementation and testing of the PBFT algorithm uses part of the code shared by the PbftSimulator project on the Github platform. Each group of experiments was run 25 times independently and averaged as the test result. The main parameter settings are shown in Table 1. In particular, in order to ensure higher consensus efficiency, the number of consensus nodes of the PBFT algorithm is selected less, usually less than 30 .

As the number of packages increases, the overall throughput of the two algorithms is increasing; when the number of packages is less than 100, the throughput of the IOT group paging random access optimization algorithm is slightly higher than that of the PBFT algorithm; when the number of packages is greater than 1000, the throughput 


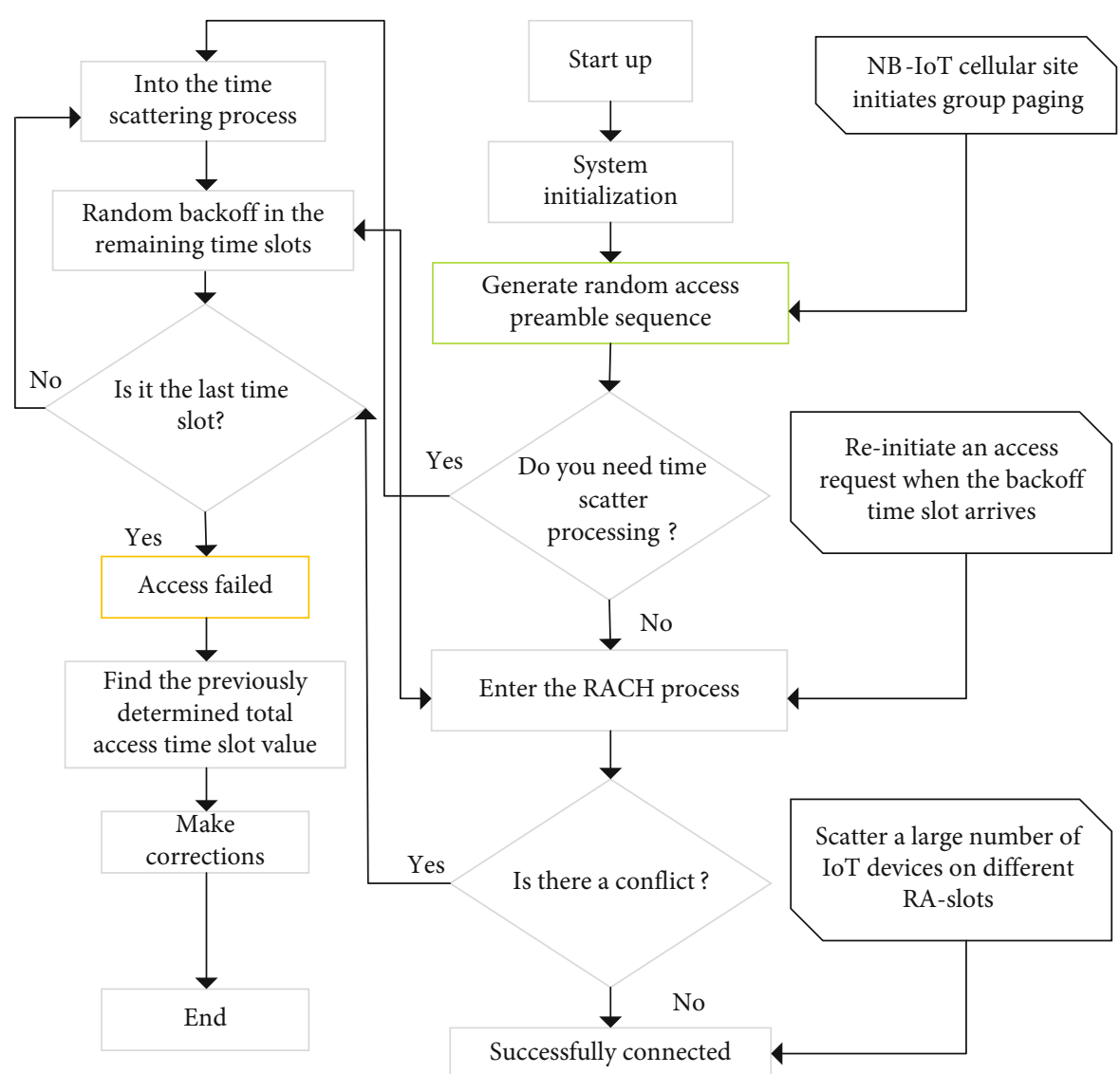

FIGURE 2: Flow chart of random access system for IoT group paging based on time slot scattering.

TABLE 1: Simulation parameter table for evaluating algorithm throughput and delay.

\begin{tabular}{lc}
\hline Simulation parameters & Values \\
\hline Maximum number of simultaneous requests & 6500 \\
Total number of request messages & $3000 \sim 8000$ \\
Malicious consensus node & 1 \\
Number of consensus nodes & 7 \\
Number of failed nodes & 1 \\
The size of the request message & 300 bytes \\
Rated bandwidth of the network between nodes & 7500 bytes \\
Basic network delay between nodes & $3 \mathrm{~ms}$ \\
Network delay disturbance range between nodes & $0.5 \mathrm{~ms}$ \\
\hline
\end{tabular}

of the IOT group paging random access optimization algorithm is significantly greater than that of the PBFT algorithm, and as the number of packages increases, the throughput difference gradually increases. Figure 3 shows the relationship between throughput and packaging quantity.

Without considering the delay, it is not rigorous and meaningless to discuss the throughput, and the delay is also an indicator to measure the responsiveness of the algorithm, so this article also tests the relationship between the delay and the number of packages. As the number of packages increases, the delays of both algorithms increase. It can be seen from Figure 4 that the time delay of the IOT group paging random access optimization algorithm is less than that of the PBFT algorithm.

As the number of packages increases, both throughput and delay increase linearly. However, in actual alliance chain applications, the delay is generally required to be less than 3 seconds. Therefore, based on practical application considerations, the discussion of the throughput and delay comparison between the algorithm in this paper and the PBFT algorithm should be limited to the range of delay less than 3 seconds. Under the simulation conditions of this article, when the number of packages is 4000 , the delay of the IOT group paging random access optimization algorithm is 2812 milliseconds, and the delay of the PBFT algorithm is 2900 milliseconds; and the throughput of the PBFT algorithm is 1379 transactions/sec. At this time, compared with the PBFT algorithm, the throughput of the IOT group paging random access optimization algorithm is increased by about $3.12 \%$, and the delay is reduced by about $3.03 \%$.

4.2. Analysis of Communication Bandwidth Overhead. In order to evaluate the communication bandwidth overhead of the IOT group paging random access optimization algorithm, on a PC configured with Intel Core i7-6700M @3.40 GH processor and $16 \mathrm{G}$ memory, installed with a 64-bit Windows7 system, the algorithms are used for mathematical calculation simulation, and the parameter settings are shown in Table 2. 

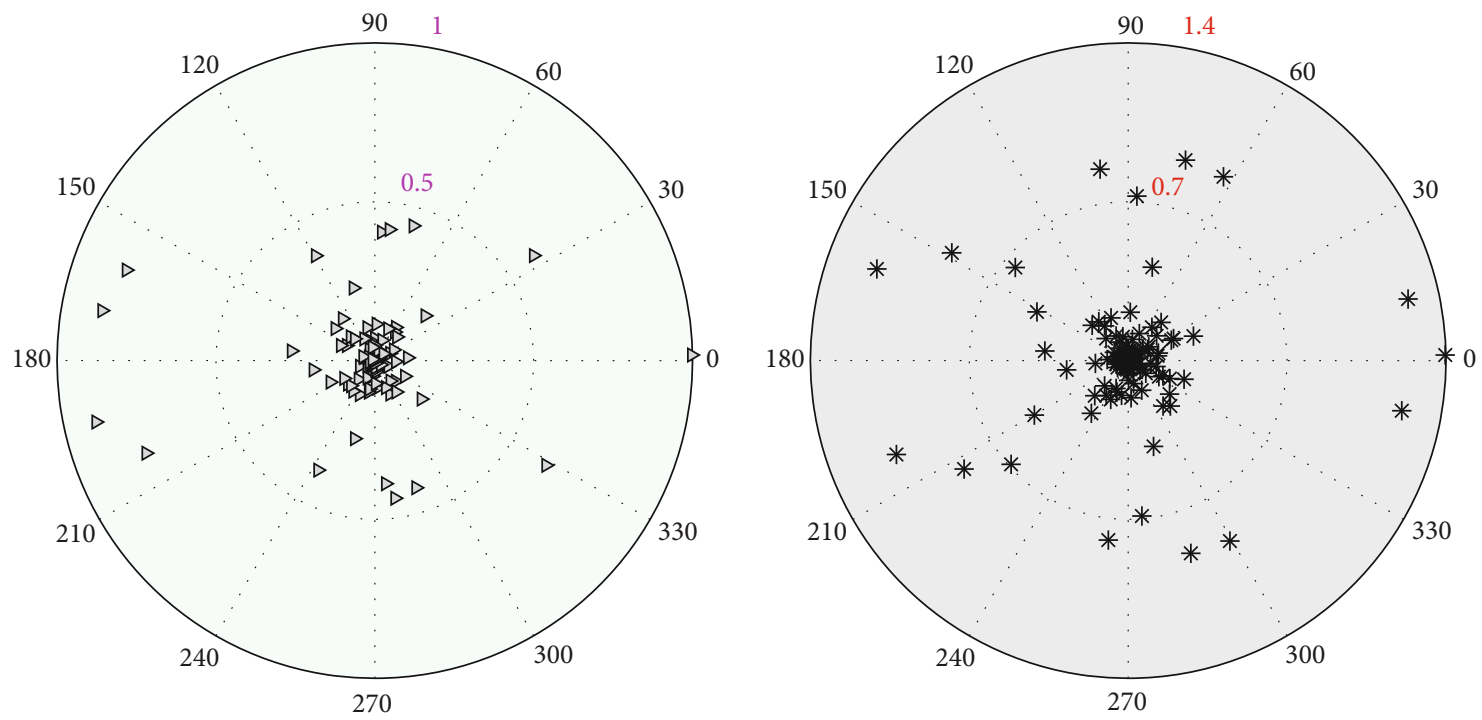

FIGURE 3: The relationship between throughput and packaging quantity. (1) PBFT algorithm. (2) IOT group paging random access optimization algorithm.

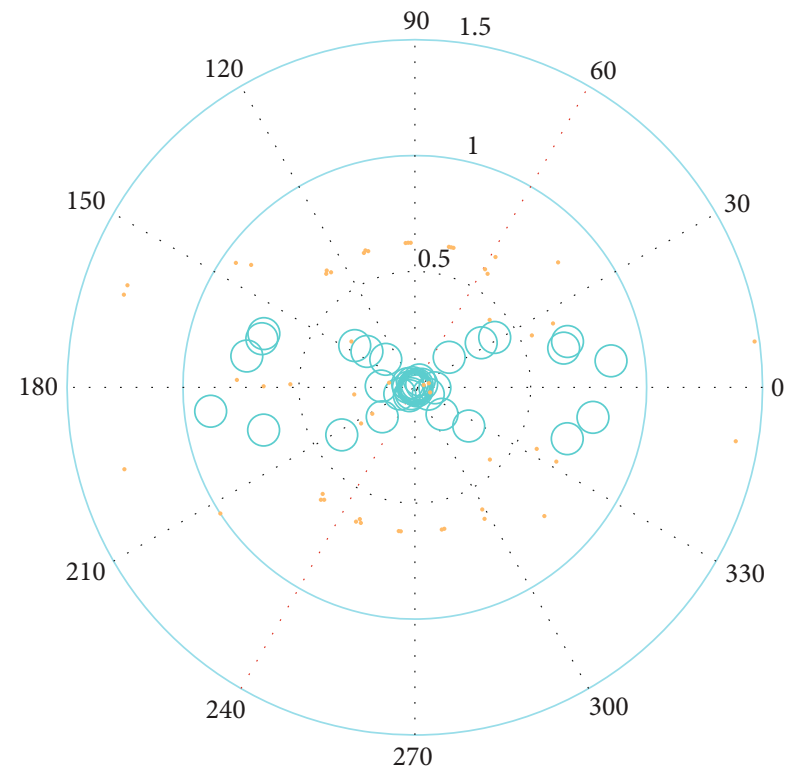

PBFT algorithm

IOT group paging random access optimization algorithm

FIGURE 4: The relationship between delay and packaging quantity.

TABLE 2: Simulation parameter table of algorithm bandwidth overhead.

\begin{tabular}{lc}
\hline Simulation parameters & Values \\
\hline Vote & $0.1 \mathrm{~KB}$ \\
$N$ & $<60$ \\
Message & $0.3 \mathrm{~KB}$ \\
$k$ & $<20$ \\
Size block & $512 \mathrm{~KB}$ \\
\hline
\end{tabular}

The first set of simulations is to compare the bandwidth overhead of different algorithms. In this experiment, the number of fixed voting nodes $N_{V}=1000$, and the number of consensus executions in a round of voting cycle is $k=5$. With the increase of the number of consensus nodes $N$, the bandwidth cost of the three algorithms increases exponentially, and the growth rate of the algorithm in this paper is small, and the growth rate of the PBFT algorithm is larger; when the number of consensus nodes $N<15$, the size relationship is that the algorithm in this paper $>$ PBFT. This is because the algorithm in this paper increases the bandwidth overhead of the election process compared with the PBFT algorithm. Under the experimental conditions of this paper, when the number of consensus nodes is 7 , this algorithm reduces the bandwidth cost of the PBFT algorithm by about $45 \%$. It can be analyzed that in the case of a large number of consensus nodes, the IOT group paging random access optimization algorithm has lower bandwidth overhead. The relationship between communication bandwidth overhead and the number of consensus nodes is shown in Figure 5.

The second set of tests is to compare the bandwidth overhead of different algorithms when applied to automated packaging systems. When applied to an automated packaging system, consensus is based on a block containing multiple packages as the smallest unit. This is different from the consensus process in the first set of experiments where one package is the smallest unit, and the test results are also different. Figures 5 and 6 have different trends. Under the simulation conditions of this article, when the number of nodes is 7 , the bandwidth overhead between the IOT group paging random access optimization algorithm and the PBFT algorithm is relatively large.

4.3. Complexity Analysis. In order to measure the growth relationship between algorithm execution efficiency and resource overhead and data scale, time complexity and space complexity are used as indicators to analyze the complexity 


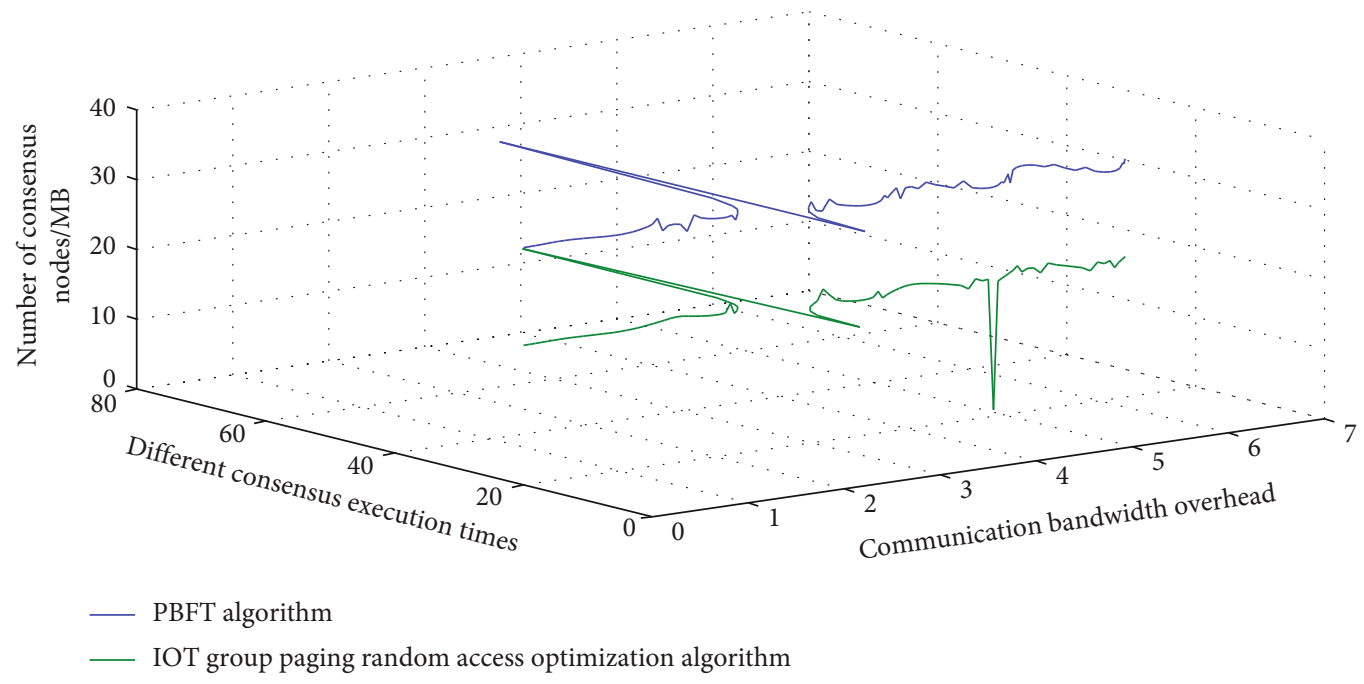

FiguRE 5: The relationship between communication bandwidth overhead and the number of consensus nodes.

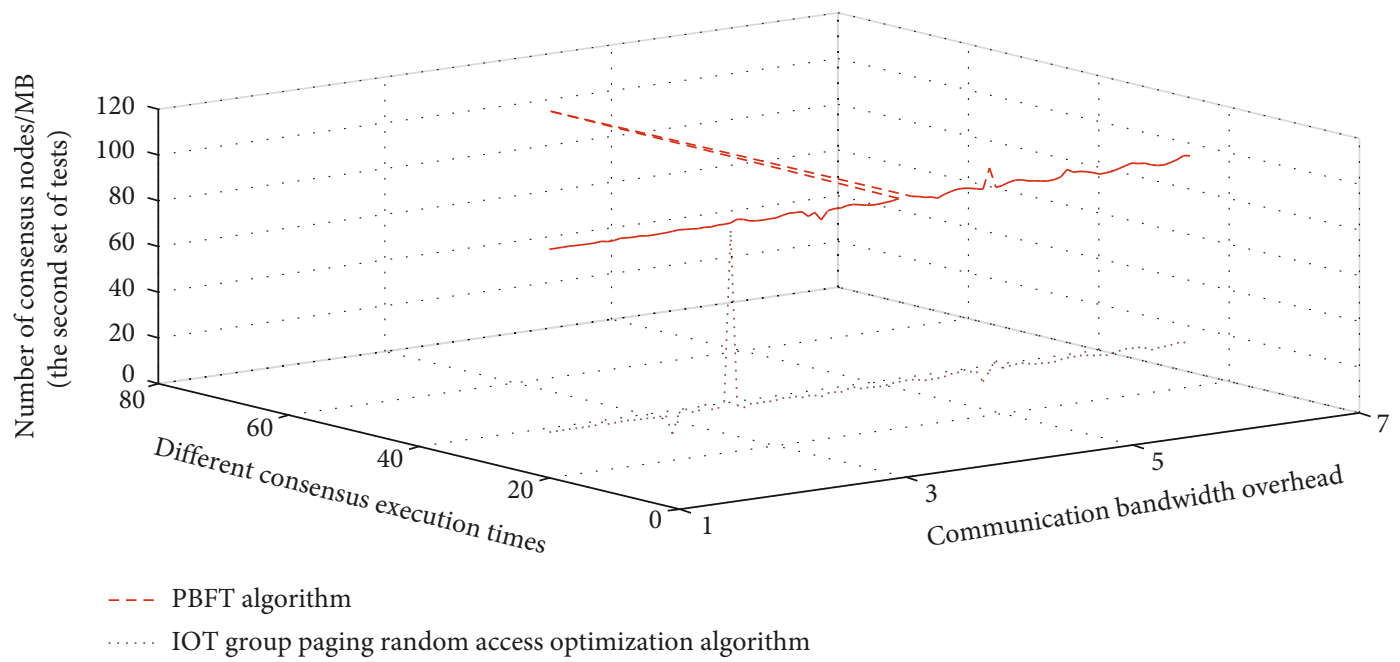

FIGURE 6: The relationship between communication bandwidth overhead and the number of consensus nodes (the second set of tests).

of the IOT group paging random access optimization algorithm.

Time complexity represents the growth relationship between the execution time of an algorithm and the size of the data and is a measure of the amount of time the algorithm occupies during its operation. For the time complexity analysis of the IOT group paging random access optimization algorithm, this section is measured by the number of basic operations performed by the algorithm under a given input scale. The IOT group paging random access optimization algorithm implements two processes of election and consensus, and one election process supports multiple rounds of consensus. The election process includes 4 stages of voting request, preparation, response, and broadcast results. The consensus process includes 4 stages of request, prepreparation, submission, and response.

Given the input size $n$, assuming that an election process supports $m(m<n)$ rounds of consensus, then the basic operation number of the algorithm in the voting request phase is 1 , the basic operation number of the algorithm in the preparation phase is $n-1$, and the operand is $n$, the basic operand of the algorithm in the broadcast phase is $n$, the basic operand of the algorithm in the request phase is $m$, the basic operand of the algorithm in the prepreparation phase is $m *(n-1)$, and the basic operand of the algorithm in the submit phase is $m * n *(n-1)$; the basic operand of the algorithm in the response phase is $m * n$.

Space complexity represents the growth relationship between the storage space of the algorithm and the data size and is a measure of the storage space temporarily occupied by the algorithm during operation. For the space complexity analysis of the IOT group paging random access optimization algorithm, this section is measured by the size of the storage space occupied by the algorithm under a given input scale. Similar to the analysis of algorithm's time complexity, 


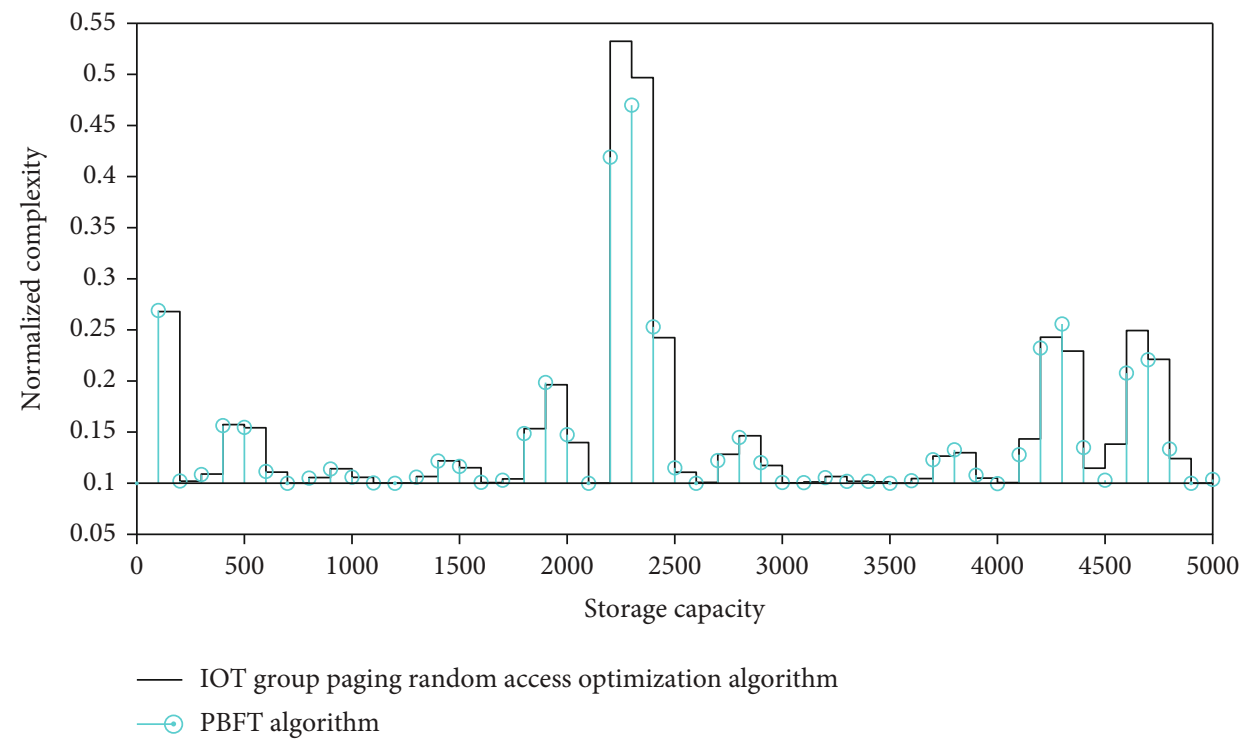

FIGURE 7: Simulation results of normalized complexity.

given the input size $n$, assuming that an election process supports $m(m<n)$ rounds of consensus, then the storage size of the voting request phase is 1 , and the storage size of the preparation phase is $n-1$; the storage size of the response phase is $n$, the storage size of the broadcast phase is $n$, the storage size of the request phase is $m$, the storage size of the pre-preparation phase is $m *(n-1)$, and the storage size of the commit phase is $m * n *(n-1)$; the storage size of the response stage is $m * n$. The stages are carried out in sequence. The simulation result of complexity normalization is shown in Figure 7. It can be seen from Figure 7 that the complexity of the IOT group paging random access optimization algorithm and the PBFT algorithm are not much different.

\section{Conclusion}

This paper studies the information interaction mechanism between the cloud service system and the mobile terminal and uses web service technology to implement the application channel for users to obtain system services and the interface design for terminal device data interaction. The information interaction mechanism between the cloud service system and the manufacturing system is studied, the Hadoop system is used to classify and mine the order information of the cloud service system, the design of the information interaction between the cloud platform and the manufacturing layer equipment is completed, and the manufacturing system layer flexibility is planned and designed. For the implementation process of automated production, the design of the cloud-assisted decision-making mechanism of the cloud service system for the production activities of the manufacturing system is designed, so as to realize the intelligent production function of the automated packaging system. In the $5 \mathrm{G}$ network architecture using NB-IoT technology, this paper proposes a random access optimization strategy based on time slot scattering for the access scenario of IoT group paging. First, a mathematical model based on queuing theory is established for the access scenario of IoT group paging, and on the basis of this model, the scattering threshold and scattering time slot of IoT devices are derived through mathematical formulas. According to the actual application scenario, the time slot scattering algorithm is modified to make it closer to the ideal value. This article builds a list of credit nodes. The credit node list has two functions. One is to improve the participation mode of consensus nodes from static to dynamic, and nodes can enter or exit dynamically; the other is to support the selection of trusted nodes by voting. During the voting period, all nodes can vote, and the top nodes with the most votes form a list of trusted nodes, which will be the candidate list of consensus nodes. This paper designs a credit evaluation mechanism. This mechanism is the follow-up of consensus node election, including credit value calculation model and node election strategy. Through this mechanism, a trusted consensus node (master node and replica node) can be elected from the list of credit nodes. Trusted consensus nodes will improve system fault tolerance and reduce communication overhead. Based on the credit evaluation mechanism, the credibility of the consensus node is ensured, thereby simplifying the three-phase protocol of the PBFT algorithm into two phases to further reduce communication overhead and algorithm delay.

\section{Data Availability}

The data used to support the findings of this study are available from the corresponding author upon request.

\section{Conflicts of Interest}

The authors declare that they have no known competing financial interests or personal relationships that could have appeared to influence the work reported in this paper. 


\section{Acknowledgments}

This research supported by "Research on interactive intelligent packaging design under the background of 5G era" (project no. 2021ky0178), which is the project to improve the basic scientific research ability of young and middle-aged teachers in colleges and universities in Guangxi in 2021.

\section{References}

[1] T. Taleb, K. Samdanis, B. Mada, H. Flinck, S. Dutta, and D. Sabella, "On multi-access edge computing: a survey of the emerging $5 \mathrm{~g}$ network edge cloud architecture \& orchestration," IEEE Communications Surveys \& Tutorials, vol. 19, no. 3, pp. 1657-1681, 2017.

[2] D. Soldani and A. Manzalini, "Horizon 2020 and beyond: on the $5 \mathrm{G}$ operating system for a true digital society," IEEE Vehicular Technology Magazine, vol. 10, no. 1, pp. 32-42, 2015.

[3] A. Al-Fuqaha, M. Guizani, M. Mohammadi, M. Aledhari, and M. Ayyash, "Internet of things: a survey on enabling technologies, protocols, and applications," IEEE Communications Surveys \& Tutorials, vol. 17, no. 4, pp. 2347-2376, 2015.

[4] S. Zhang and T. N. Wong, "Integrated process planning and scheduling: an enhanced ant colony optimization heuristic with parameter tuning," Journal of Intelligent Manufacturing, vol. 29, no. 3, pp. 585-601, 2018.

[5] J. A. Warren, M. E. Riddle, D. J. Graziano et al., "Energy impacts of wide band gap semiconductors in U.S. light-duty electric vehicle fleet," Environmental science \& technology, vol. 49, no. 17, pp. 10294-10302, 2015.

[6] J. Moura and D. Hutchison, "Game theory for multi-access edge computing: survey, use cases, and future trends," IEEE Communications Surveys \& Tutorials, vol. 21, no. 1, pp. 260288, 2019.

[7] S. A. A. Shah, E. Ahmed, M. Imran, and S. Zeadally, "5G for vehicular communications," IEEE Communications Magazine, vol. 56, no. 1, pp. 111-117, 2018.

[8] K. S. E. Phala, A. Kumar, and G. P. Hancke, "Air quality monitoring system based on ISO/IEC/IEEE 21451 standards," IEEE Sensors Journal, vol. 16, no. 12, pp. 5037-5045, 2016.

[9] S.-Y. Wang and L. Wang, "An estimation of distribution algorithm-based memetic algorithm for the distributed assembly permutation flow-shop scheduling problem," IEEE Transactions on Systems, Man, and Cybernetics: Systems, vol. 46, no. 1, pp. 139-149, 2016.

[10] X. She, A. Q. Huang, O. Lucia, and B. Ozpineci, "Review of silicon carbide power devices and their applications," IEEE Transactions on Industrial Electronics, vol. 64, no. 10, pp. 8193-8205, 2017.

[11] M. Mehrabi, D. You, V. Latzko, H. Salah, M. Reisslein, and F. H. P. Fitzek, "Device-enhanced mec: multi-access edge computing (mec) aided by end device computation and caching: a survey," IEEE Access, vol. 7, pp. 166079-166108, 2019.

[12] R. Zhang, Z. Zhong, J. Zhao, B. Li, and K. Wang, "Channel measurement and packet-level modeling for V2I spatial multiplexing uplinks using massive MIMO," IEEE Transactions on Vehicular Technology, vol. 65, no. 10, pp. 7831-7843, 2016.

[13] A. Ali, G. A. Shah, M. O. Farooq, and U. Ghani, "Technologies and challenges in developing machine-to-machine applications: a survey," Journal of Network and Computer Applications, vol. 83, no. 4, pp. 124-139, 2017.
[14] X.-L. Zheng and L. Wang, "A collaborative multiobjective fruit fly optimization algorithm for the resource constrained unrelated parallel machine green scheduling problem," IEEE Transactions on Systems, Man, and Cybernetics: Systems, vol. 48, no. 5, pp. 790-800, 2018.

[15] C. Chen, F. Luo, and Y. Kang, "A review of sic power module packaging: layout, material system and integration," CPSS Transactions on Power Electronics and Applications, vol. 2, no. 3, pp. 170-186, 2017.

[16] K. Zhang, S. Leng, Y. He, S. Maharjan, and Y. Zhang, "Mobile edge computing and networking for green and low-latency internet of things," IEEE Communications Magazine, vol. 56, no. 5, pp. 39-45, 2018.

[17] K. Liu, J. K. Y. Ng, V. C. S. Lee, S. H. Son, and I. Stojmenovic, "Cooperative data scheduling in hybrid vehicular ad hoc networks: VANET as a software defined network," IEEE/ACM transactions on networking, vol. 24, no. 3, pp. 1759-1773, 2016.

[18] M. Centenaro, L. Vangelista, A. Zanella, and M. Zorzi, "Longrange communications in unlicensed bands: the rising stars in the IoT and smart city scenarios," IEEE Wireless Communications, vol. 23, no. 5, pp. 60-67, 2016.

[19] G. Mejia, J. P. Caballero-Villalobos, and C. Montoya, "Petri nets and deadlock-free scheduling of open shop manufacturing systems," IEEE Transactions on Systems, Man, and Cybernetics: Systems, vol. 48, no. 6, pp. 1017-1028, 2018.

[20] S. Ji, Z. Zhang, and F. Wang, "Overview of high voltage sic power semiconductor devices: development and application," CES Transactions on Electrical Machines and Systems, vol. 1, no. 3, pp. 254-264, 2017.

[21] P. Kourouthanassis, C. Boletsis, C. Bardaki, and D. Chasanidou, "Tourists responses to mobile augmented reality travel guides: the role of emotions on adoption behavior," Pervasive and Mobile Computing, vol. 18, pp. 71-87, 2015.

[22] T. Qiu, X. Wang, C. Chen, M. Atiquzzaman, and L. Liu, "TMED: a spider web-like transmission mechanism for emergency data in vehicular ad hoc networks," IEEE Transactions on Vehicular Technology, vol. 67, no. 9, pp. 8682-8694, 2018.

[23] M. Ndiaye, G. P. Hancke, and A. M. Abu-Mahfouz, "Software defined networking for improved wireless sensor network management: a survey," Sensors, vol. 17, no. 5, pp. 1031-1032, 2017.

[24] M. Gen, W. Zhang, L. Lin, and Y. Yun, "Recent advances in hybrid evolutionary algorithms for multiobjective manufacturing scheduling," Computers and Industrial Engineering, vol. 112, pp. 616-633, 2017.

[25] C. Durand, M. Klingler, D. Coutellier, and H. Naceur, "Power cycling reliability of power module: a survey," IEEE Transactions on Device and Materials Reliability, vol. 16, no. 1, pp. 80-97, 2016.

[26] J. Pan and J. McElhannon, "Future edge cloud and edge computing for internet of things applications," IEEE Internet of Things Journal, vol. 5, no. 1, pp. 439-449, 2018.

[27] T. Qiu, R. Qiao, and D. O. Wu, "EABS: an event-aware backpressure scheduling scheme for emergency internet of things," IEEE Transactions on Mobile Computing, vol. 17, no. 1, pp. 7284, 2018.

[28] C. Chen, Y. Chen, Y. Li, Z. Huang, T. Liu, and Y. Kang, "An sicbased half-bridge module with an improved hybrid packaging method for high power density applications," IEEE Transactions on Industrial Electronics, vol. 64, no. 11, pp. 89808991, 2017. 